\title{
DENSITIES FOR RANKS OF CERTAIN PARTS \\ OF $p$-CLASS GROUPS
}

\author{
FRANK GERTH III
}

\begin{abstract}
Let $K$ be a Galois extension of the field of rational numbers of prime degree $p$, and let $C_{K}$ be the $p$-class group of $K$. In this paper densities for the ranks of certain parts of such $C_{K}$ are calculated, and these densities suggest a way to extend conjectures of Cohen and Lenstra.
\end{abstract}

1. Introduction. Let $p$ be a prime number, and let $\mathbf{Q}$ denote the field of rational numbers. Let $K$ be a Galois extension of $\mathbf{Q}$ such that $\mathrm{Gal}(K / \mathbf{Q})$ is a cyclic group of order $p$. Let $C_{K}$ denote the $p$-class group of $K$; i.e., the Sylow $p$-subgroup of the ideal class group of $K$. (For $p=2$, we shall be using the Sylow 2-subgroup of the narrow ideal class group of $K$.) Let $\sigma$ be a generator of $\operatorname{Gal}(K / \mathbf{Q})$, and let $C_{K}^{(1-\sigma)^{i}}=\left\{a^{(1-\sigma)^{\prime}}: a \in C_{K}\right\}$ for $i=0,1,2, \ldots$ Suppose exactly $t$ primes ramify in $K / \mathbf{Q}$. It is a classical result that $C_{K} / C_{K}^{1-\sigma}$ is an elementary abelian $p$-group with rank equal to $t-1$. Furthermore, $C_{K}^{(1-\sigma)^{i}} / C_{K}^{(1-\sigma)^{i+1}}$ is an elementary abelian $p$-group of each $i$, and

$$
\operatorname{rank} C_{K}=\operatorname{rank}\left(C_{K} / C_{K}^{p}\right)=\sum_{i=1}^{p-1} \operatorname{rank}\left(C_{K}^{(1-\sigma)^{\prime \cdots 1}} / C_{K}^{(1-\sigma)^{\prime}}\right),
$$

where $C_{K}^{p}=\left\{a^{p}: a \in C_{K}\right\}$ (cf. [9, Proposition 4.2 and 11, Satz 6]). Since we know that rank $C_{K} / C_{K}^{1-\sigma}=t-1$, we shall focus our attention on $C_{K}^{1-\sigma} / C_{K}^{(1-\sigma)^{2}}$. If we let $R_{K}=\operatorname{rank}\left(C_{K}^{1-\sigma} / C_{K}^{(1-\sigma)^{2}}\right)$, then $0 \leqslant R_{K} \leqslant t-1$. In this paper we shall consider the following question: how likely is $R_{K}=0, R_{K}=1, R_{K}=2$, etc., as $t \rightarrow \infty$.

2. Statement of main results. Let notation be the same as in $\S 1$. For each positive integer $t$, each nonnegative integer $r$, and each positive real number $x$, we define

$$
A_{t}=\text { \{cyclic extensions } K \text { of } \mathbf{Q} \text { of degree } p \text { with exactly } t \text { ramified primes }
$$

(when $p=2$, we shall consider separately the imaginary and real quadratic fields)

$$
\begin{aligned}
A_{t: x} & =\left\{K \in A_{t}: \text { the conductor of } K \text { is } \leqslant x\right\}, \\
A_{t, r ; x} & =\left\{K \in A_{t: x}: R_{K}=r\right\} .
\end{aligned}
$$

Then we define the density $d_{t, r}$ by

$$
d_{t, r}=\lim _{x \rightarrow \infty} \frac{\left|A_{t, r ; x}\right|}{\left|A_{t ; x}\right|}
$$

Received by the editors March 25, 1985 and, in revised form, November 25, 1985.

1980 Mathematics Subject Classification (1985 Revision). Primary 11R20, 11R29, 11R45. 
where $|S|$ denotes the cardinality of a set $S$. We then define the limit density $d_{\infty, r}$ by

$$
d_{\infty, r}=\lim _{t \rightarrow \infty} d_{t, r}
$$

Our theorems will show that these limits exist and will provide the values for these limits. For $p=2$, we have obtained the following result in [6, Theorems 4.3 and 5.11].

THEOREM 1. For imaginary quadratic fields,

$$
d_{\infty, r}=\frac{2^{-r^{2}} \prod_{k=1}^{\infty}\left(1-2^{-k}\right)}{\prod_{k=1}^{r}\left(1-2^{-k}\right)^{2}} \text { for } r=0,1,2, \ldots
$$

For real quadratic fields,

$$
d_{\infty, r}=\frac{2^{-r(r+1)} \prod_{k=1}^{\infty}\left(1-2^{-k}\right)}{\prod_{k=1}^{r}\left(1-2^{-k}\right) \prod_{k=1}^{r+1}\left(1-2^{-k}\right)} \quad \text { for } r=0,1,2, \ldots
$$

REMARK. When $p=2$,

$$
R_{K}=\operatorname{rank}\left(C_{K}^{1-\sigma} / C_{K}^{(1-\sigma)^{2}}\right)=\operatorname{rank}\left(C_{K}^{2} / C_{K}^{4}\right)=4 \text {-class rank of } C_{K} .
$$

So Theorem 1 gives limit densities for the 4-class ranks of imaginary and real quadratic fiels.

Our goal in this paper is to prove the following theorem.

THEOREM 2. Suppose $p \geqslant 3$. Then

$$
d_{\infty, r}=\frac{p^{-r(r+1)} \prod_{k=1}^{\infty}\left(1-p^{-k}\right)}{\prod_{k=1}^{r}\left(1-p^{-k}\right) \prod_{k=1}^{r+1}\left(1-p^{-k}\right)} \quad \text { for } r=0,1,2, \ldots .
$$

Values for $d_{\infty, r}$ for small $p$ and $r$ appear in the Appendix.

3. Proof of Theorem 2. We let notation be the same as in $\$ \S 1$ and 2 , and we assume $p \geqslant 3$. First we note that the fields $K$ in $A_{t: x}$ have conductor $f_{K}=p^{2} p_{1} \cdots$ $p_{t-1}$ or $f_{K}=p_{1} \cdots p_{t}$, where $p_{1}, \ldots, p_{t}$ are distinct rational primes with each $p_{i} \equiv 1$ $(\bmod p)$. We can ignore the fields $K$ with $f_{K}=p^{2} p_{1} \cdots p_{t-1} \leqslant x$ when calculating $d_{t, r}$ since the number of such fields is

$$
O\left(\frac{x(\log \log x)^{t-2}}{\log x}\right), \quad \text { while }\left|A_{t: x}\right| \gg \frac{x(\log \log x)^{t-1}}{\log x}
$$

(cf. [10, Theorem 437 and 4, p. 201]). For each field $K$ with $f_{K}=p_{1} \cdots p_{t}$, we introduce a $t \times t$ matrix $M_{K}$ whose entries $m_{i j}$ are defined in terms of Hilbert symbols by

$$
\omega^{m_{t \prime}}=\left(\frac{p_{j}, \mu_{K}}{\wp_{i}}\right) \text { for } 1 \leqslant i \leqslant t, 1 \leqslant j \leqslant t,
$$

where $\omega$ is a primitive $p$ th root of unity, $\wp_{i}$ is a prime of $F=\mathbf{Q}(\omega)$ above $p_{i}$, and $\mu_{K}$ is an element of $F$ satisfying $K F=F\left(\mu_{K}^{1 / p}\right)$. (See [3, p. 197] for more details.) We view $M_{K}$ as a matrix over $\mathbf{F}_{p}$, the finite field with $p$ elements. It is known that $R_{K}=t-1-\operatorname{rank} M_{K}$ (cf. [9, Proposition 4.6, Proposition 4.7, and IV B 4, p. 45]). 
Using this fact, we have in effect determined $d_{t, r}$ in [5]. To be more precise, $d_{t, r}$ in (1) corresponds to $B_{t, e}$ in [5, Equation 2.2]. So

$$
d_{t, r}=\left[\prod_{j=1}^{t-1-r}\left(1-\frac{1}{p^{t+1-j}}\right)\right] \cdot \frac{1}{p^{t r}} \cdot \sum_{\substack{i_{1}+\cdots+i_{t-1, r} \leqslant r \\ \text { each } i_{s} \geqslant 0}}\left(\prod_{s=1}^{t-1-r} p^{s i_{s}}\right) .
$$

(When $r=t-1, d_{t, t-1}=p^{-t(t-1)}$.) The main ideas used in proving (3) can be explained as follows. Let $J$ be any $t \times t$ matrix with coefficients in $\mathbf{F}_{p}$ and with the sum of the entries in each column of $J$ equal to 0 . Let

$$
N_{J}(x)=\mid\left\{K: M_{K}=J \text { and } f_{K} \leqslant x\right\} \mid .
$$

Then $N_{J}(x)=h(x)+o(h(x))$, where $h(x)$ is a function that is independent of $J$. (This corresponds to equidistribution of the Hilbert symbols. See [3, p. 196 and pp. 200-206] for more details.) It follows that

$$
\frac{\left|A_{t, r: x}\right|}{\left|A_{t: x}\right|}=\frac{\sum_{J}^{(r)} N_{J}(x)}{\sum_{J} N_{J}(x)}=\frac{\sum_{J}^{(r)} 1+o(1)}{\sum_{J} 1+o(1)}
$$

where $\sum_{J}^{(r)}$ denotes a sum over those $J$ with rank $J=t-1-r$. Hence

$$
d_{t, r}=\left(\sum_{J}^{(r)} 1\right) /\left(\sum_{J} 1\right)
$$

This rational number is calculated in [5] and is given by (3).

We must show that $\lim _{t \rightarrow \infty} d_{t, r}$ has the value given by Theorem 2 . We let $k=t+1-j$ and $w=t-1-r$. Then

$$
\begin{aligned}
& d_{t, r}=\left[\prod_{k=r+2}^{t}\left(1-\frac{1}{p^{k}}\right)\right] \cdot \frac{1}{p^{r(r+1)} p^{w r}} \cdot \sum_{\substack{i_{1}+\cdots+i_{w} \leqslant r \\
\text { each } i_{s} \geqslant 0}} p^{1 i_{1}+2 i_{2}+\cdots+w i_{w}} \\
& =\left[\frac{\prod_{k=1}^{t}\left(1-p^{-k}\right)}{\prod_{k=1}^{r+1}\left(1-p^{-k}\right)}\right] \cdot p^{-r(r+1)} \cdot\left[\frac{1}{p^{w r}} \sum_{\substack{i_{1}+\cdots+i_{w} \leqslant r \\
\text { each } i_{s} \geqslant 0}} p^{1 i_{1}+2 i_{2}+\cdots+n i_{w}}\right],
\end{aligned}
$$

and then

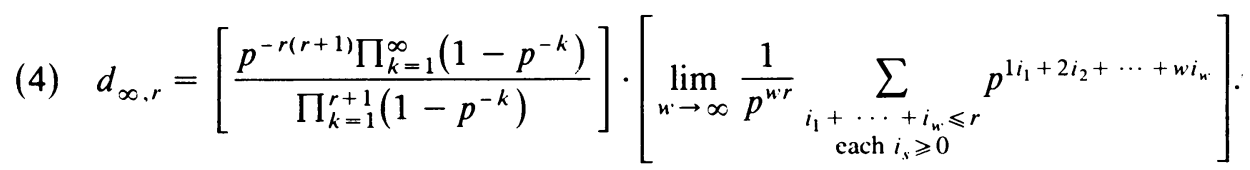
If $r=0$, then $d_{\infty, 0}$ in (4) is the same as $d_{\infty, 0}$ in the statement of Theorem 2. So we may assume $r \geqslant 1$. To evaluate the limit in (4), we shall use the following lemma.

LeMma. Let $w$ and $m$ be positive integers, and let

$$
F_{w, m}=\frac{1}{p^{w m}} \sum_{\substack{i_{1}+\cdots+i_{w=m}=m \\ \text { each } i_{s} \geqslant 0}} p^{1 i_{1}+2 i_{2}+\cdots+w i_{w}}
$$

Then

$$
\lim _{n \rightarrow \infty} F_{w, m}=\prod_{k=1}^{m}\left(1-p^{-k}\right)^{-1}
$$


Proof. First we note that

$$
F_{n^{\prime}, m}=\sum_{\substack{i_{1}+\cdots+i_{w}=m \\ \text { each } i_{1} \geqslant 0}} p^{\left(1-w^{\prime}\right) i_{1}+\left(2-n^{\prime}\right) i_{2}+\cdots+(-1) i_{w} 1_{1}+0 i_{w}}
$$

since $1 / p^{n \cdot m}=p^{-n^{\prime}\left(i_{1}+\cdots+i_{n^{\prime}}\right)}$. Also we note that $F_{n, m}$ appears in $F_{u^{\prime}+1, m}$ exactly as those terms having $i_{1}=0$. Then

$$
\lim _{n \rightarrow \infty} F_{n \cdot m}=\sum_{l=0}^{\infty} b_{l, m} p^{-l},
$$

where $b_{l, m}$ is the number of times that

$$
l=(w-1) i_{1}+(w-2) i_{2}+\cdots+1 i_{n-1}+0 i_{n} \text {. for some } w .
$$

Since $i_{1}+\cdots+i_{n^{-1}} \leqslant m$, such an expression can be associated to a partition of $l$ into at most $m$ parts. Conversely, given such a partition, we can let $w-1$ be the largest integer appearing in it and let $i_{s}$ be the number of times $w-s$ appears, $1 \leqslant s \leqslant w-1$. So $b_{l, m}$ is the number of partitions of $l$ into at most $m$ parts. Next we observe that

$$
\begin{aligned}
\prod_{k=1}^{m}\left(1-p^{-k}\right)^{-1} & =\prod_{k=1}^{m}\left(1+p^{-k}+p^{-2 k}+\cdots\right) \\
& =\sum_{j_{1}, j_{2}, \ldots, j_{m} \geqslant 0} p^{-1 j_{1}-2 j_{2}-\cdots-m j_{m}}=\sum_{l=0}^{\infty} c_{l, m l} p^{-l},
\end{aligned}
$$

where $c_{l, m}$ is the number of times that $l=1 j_{1}+2 j_{2}+\cdots+m j_{m}$. But then $c_{l, m}$ is the number of partitions of $l$ into parts with each part at most $m$. From [10, Theorem 343], $b_{l, m}=c_{l, m}$ for all $l$ and $m$, and hence the lemma is proved.

Now applying the Lemma to the sum in (4), we get

$$
\begin{aligned}
\lim _{n \rightarrow \infty} \frac{1}{p^{n \cdot r}} \sum_{\substack{i_{1}+\cdots+i_{n} \leqslant r \\
\text { each } i_{s} \geqslant 0}} p^{1 i_{1}+2 i_{2}+\cdots+n \cdot i_{n}} & =\lim _{n \rightarrow \infty}\left[\frac{1}{p^{n \cdot r}}+\sum_{m=1}^{r} \frac{1}{p^{n \cdot(r-m)}} F_{n^{\prime}, m}\right] \\
& =\lim _{n \rightarrow \infty} F_{w, r}=\prod_{k=1}^{r}\left(1-p^{-k}\right)^{-1},
\end{aligned}
$$

which completes the proof of Theorem 2 .

4. Cohen-Lenstra Conjectures. We let notation be the same as in previous sections. In [1] Cohen and Lenstra have made various conjectures that apply to the prime to $p$ part of the class groups for Galois extensions of $\mathbf{Q}$ of degree $p$. Since our results apply to the $p$ part of the class groups, our results do not prove or disprove any of the Cohen-Lenstra Conjectures. However, our results do have an interesting relationship with the Cohen-Lenstra Conjectures. To describe this relationship, we first let $S_{K}$ be the narrow ideal class group of $K$, and we let $H_{K}=S_{K}^{1-\sigma}$, which is the narrow principal genus of $K$ for the fields $K$ we are considering. Then our Theorems 1 and 2 appear to be what would be predicated if we assumed that Fundamental Assumptions 8.1 and Theorem 6.3 in [1] apply to $H_{K}$. Actually the appropriate Cohen-Lenstra 
probability is defined in a different way than our density $d_{\infty, r}$. More precisely, let

$$
d_{r}=\lim _{x \rightarrow \infty}\left(\sum_{\substack{K \\\left|D_{K}\right| \leqslant x \\ R_{K}=r}} 1 / \sum_{\substack{K \\\left|D_{K}\right| \leqslant x}} 1\right)
$$

where $K$ ranges over the Galois extensions of $\mathbf{Q}$ of degree $p, D_{K}$ is the discriminant of $K$, and $R_{K}$ is defined in $\S 1$. (When $p=2$, the real and imaginary quadratic fields are handled separately.) This Cohen-Lenstra probability $d_{r}$ omits all reference to the number $t$ of ramified primes and deals with the discriminant $D_{K}$ instead of the conductor $f_{K}$. Since $\left|D_{K}\right|=f_{K}^{-1}$, there is no difficulty in passing from the conductor to the discriminant. So we see that

$$
d_{r}=\lim _{x \rightarrow \infty}\left(\sum_{s=1}^{\infty}\left|A_{s, r: x}\right| / \sum_{s=1}^{\infty}\left|A_{s: x}\right|\right) .
$$

(Note that for each $x$ the above sums are finite.) However,

$$
d_{\infty, r}=\lim _{t \rightarrow \infty} d_{t, r}=\lim _{t \rightarrow \infty}\left(\lim _{x \rightarrow \infty} \frac{\left|A_{t, r: x}\right|}{\left|A_{t: x}\right|}\right) .
$$

Since for fixed $t$ and $s<t,\left|A_{s, r: x}\right|=o\left(\left|A_{t, r: x}\right|\right)$ and $\left|A_{s: x}\right|=o\left(\left|A_{t: x}\right|\right)$ as $x \rightarrow \infty$ (cf. [5, Propositions 3.3 and 3.4 and 6, Propositions 2.1 and 5.1]), then

$$
d_{\infty, r}=\lim _{t \rightarrow \infty}\left(\lim _{x \rightarrow \infty} \frac{\sum_{s=1}^{t}\left|A_{s, r: x}\right|}{\sum_{s=1}^{t}\left|A_{s: x}\right|}\right) .
$$

From (5) and (6), it seems plausible that $d_{r}=d_{x, r}$, although a proof would involve more detailed estimates with explicit dependence on $t$ carefully analyzed.

Now assuming $d_{r}=d_{\infty, r}$, our results suggest that the Cohen-Lenstra Conjectures should be extended to include all of the narrow principal genus for Galois extensions of $\mathbf{Q}$ of prime degree $p$. In particular, the conjectures in $\$ 9$ of [1] could be extended to all of the narrow principal genus. As an example we mention how conjecture (C14) in [1] could be extended.

Conjecture $\left(\mathrm{C}^{\prime} 4^{\prime}\right)$. For totally real Galois extensions of $\mathbf{Q}$ of prime degree $p$ (including $p=2$ ), the probability $Z(p)$ that the narrow principal genus is trivial is given by

$$
Z(p)=\prod_{k=2}^{\infty}\left(\zeta_{\mathbf{Q}(\sqrt[p]{1})}(k)\right)^{-1}
$$

where $\zeta_{\mathbf{Q}(\sqrt[p]{1})}(s)$ is the Dedekind zeta function of the cyclotomic field $\mathbf{Q}(\sqrt[p]{1})$.

Some numerical values of $Z(p)$ are as follows: $Z(2)=0.436, Z(3)=0.714$, $Z(5)=0.903$, and $Z(7)=0.929$. Also $\lim _{p \rightarrow \infty} Z(p)=1$ (cf. [1, p. 58]). So for large $p$, one should expect the narrow principal genus to be trivial.

REMARK. The Cohen-Lenstra Conjectures should also apply to the usual principal genus, not just the narrow principal genus (cf. [6, p. 491]). 
5. Estimate of a character sum. Our proof of Theorem 2 depends on results from [ 3 and 5]. In the proof of Lemma 3 in [3], we used a certain character sum estimate (see bottom of p. 202 in [3]) that was derived in a preliminary version of [7], but this particular character sum estimate was not included in the final version of [7]. So for the sake of completeness, we sketch a proof of that character sum estimate.

The basic reference for the techniques for this character sum estimate is [2]. We suppose that $\lambda$ is a nonprincipal Dirichlet character with exponent $l$ and conductor $p_{1} \cdots p_{s}$, where $l$ is a prime and $p_{1}, \ldots, p_{s}$ are distinct primes. We let $x$ be a large real number, $q=p_{1} \cdots p_{s}, y=x / q$, and

$$
z=\exp [(\log x) /(b \log \log x)]
$$

where $b$ is a constant to be specified later. We assume $q \leqslant z$. We want to show

$$
\sum_{p \leqslant y} \lambda(p)=O\left(y /(\log q y)^{2}\right)
$$

where the sum ranges over all primes $p \leqslant y$. Note that we need only estimate $\sum_{(q .1)^{1 / 2}<p \leqslant y} \lambda(p)$ since $q \leqslant z$ and $y=x / q$ imply $(q y)^{1 / 2}=O\left(y /(\log q y)^{2}\right)$. Now

$$
\begin{aligned}
\sum_{(q .)^{1 / 2}<p \leqslant y} \lambda(p) & =\sum_{\substack{(q .)^{1 / 2}<p^{m} \leqslant y \\
m \geqslant 1}} \frac{\lambda\left(p^{m}\right) \log p}{\log \left(p^{m}\right)}-\sum_{\substack{(q .)^{1 / 2}<p^{m} \leqslant y \\
m \geqslant 2}} \frac{\lambda\left(p^{m}\right) \log p}{\log \left(p^{m}\right)} \\
& =\sum_{(q .)^{1 / 2}<n \leqslant y} \frac{\lambda(n) \Lambda(n)}{\log n}+O\left(y^{1 / 2}\right)
\end{aligned}
$$

where

$$
\Lambda(n)= \begin{cases}\log p & \text { if } n \text { is a power of a prime } p, \\ 0 & \text { otherwise. }\end{cases}
$$

Using partial summation (cf. [10, Theorem 421]), we see that (7) will be proved if we can show that

$$
\sum_{n \leqslant y} \lambda(n) \Lambda(n)=O(y /(\log q y))
$$

From [2, p. 126], we have

$$
\sum_{n \leqslant y} \lambda(n) \Lambda(n)=-y^{\beta} / \beta+R(y, T)
$$

where

$$
\begin{gathered}
|R(y, T)| \ll y(\log q y)^{2} \exp [-c(\log y) /(\log q T)] \\
+y T^{-1}(\log q y)^{2}+y^{1 / 4}(\log y) .
\end{gathered}
$$

In formulas (9) and (10), $T$ is a parameter we are free to choose; $q$ is the conductor of $\lambda$; and $c$ is a positive absolute constant. The term with $y^{\beta}$ in (9) can occur only if $\lambda$ is an "exceptional" real characer (and hence $l=2$ ). If $\lambda$ is an exceptional character, then (8) may not be valid. However, we do know that

$$
\beta<1-c_{1} / q^{1 / 2}(\log q)^{2}
$$


for some positive absolute constant $c_{1}$ (see [2, p. 99]). Then one can show that $y^{\beta}=O\left(x /(\log x)^{1+\gamma}\right)$ for some $\gamma>0$. When sums are subsequently taken over the conductors $q=p_{1} \cdots p_{s}$, fortunately the exceptional conductors are rather sparse. If these exceptional conductors are $q_{0}<q_{1}<q_{2}<\cdots$, then $q_{j+1}>q_{j}^{2}$ for each $j$ (see [2, p. 98]), and so $q_{j}>\exp \left(2^{j}\right)$ for each $j$. Since $q_{j} \leqslant \exp (\log x /(b \log \log x)$ ), then $j=O(\log \log x)$, and hence the total contribution of all $y^{\beta} / \beta$ can be incorporated into the final error term $o\left(x(\log \log x)^{s} /(\log x)\right)(\mathrm{cf}$. Lemma 3 of [3]).

It remains to show that $|R(y, T)| \ll y /(\log q y)$. By choosing $T=(\log q y)^{3}$, we see that the second and third terms on the right side of $(10)$ are $\ll y /(\log q y)$. Now

$$
\frac{y(\log q y)^{2}}{\exp \left[\frac{c(\log y)}{\log q T}\right]} \ll \frac{y(\log q y)^{2}}{\exp \left[\frac{c \log \left((q y)^{1-\delta}\right)}{(\log q y) /(b(\log \log q y))+3(\log \log q y)}\right]}
$$

for any $0<\delta<1$. We let $\varepsilon$ satisfy $0<\varepsilon<(1 / 3) c(1-\delta)$. We choose $y$ large enough so that

$$
3(\log \log q v)<\varepsilon(\log q y) /(\log \log q y),
$$

and we choose $b>0$ so that $c(1-\delta) \geqslant 3((1 / b)+\varepsilon)$. Then

$$
y(\log q y)^{2} \exp [-c(\log y) /(\log q T)] \ll y /(\log q y),
$$

and hence $|R(y, T)| \ll y /(\log q y)$.

In [3], where $l \geqslant 3,(7)$ is needed for the slightly more general case of Hecke characters over $\mathbf{Q}(\exp (2 \pi i / l))$ instead of Dirichlet characters. However, the methods are essentially the same as those used for Dirichlet characters (e.g., compare the methods in Chapter 14 of [8] with the methods in [2]). Furthermore there are no exceptional characters when $l \geqslant 3$.

ACKNOWLEDGMENT. The author thanks the referee for several helpful suggestions.

APPENDIX. Some values for $d_{\infty, r}$ in Theorems 1 and 2 are given below.

\begin{tabular}{|l|cccccc}
\hline$p$ & $r$ & 0 & 1 & 2 & 3 & 4 \\
\hline 2 (imag. case) & 0.288788 & 0.577576 & 0.128350 & 0.005239 & $4.7 \times 10^{-5}$ \\
2 (real case) & 0.577576 & 0.385051 & 0.036672 & 0.000699 & $3.0 \times 10^{-6}$ \\
3 & 0.840189 & 0.157535 & 0.002272 & $3.3 \times 10^{-6}$ & $5.1 \times 10^{-10}$ \\
5 & 0.950416 & 0.049501 & $8.3 \times 10^{-5}$ & $5.4 \times 10^{-9}$ & $1.4 \times 10^{-14}$ \\
7 & 0.976261 & 0.023729 & $1.0 \times 10^{-5}$ & $8.6 \times 10^{-11}$ & $1.5 \times 10^{-17}$
\end{tabular}

\section{REFERENCES}

1. H. Cohen and H. Lenstra, Jr., Heuristics on class groups of number fields, Lecture Notes in Math., vol. 1068, Springer-Verlag, Berlin and New York, 1984, pp. 33-62.

2. H. Davenport, Multiplicative number theory, Markham, Chicago, Ill., 1967.

3. F. Gerth, Counting certain number fields with prescribed l-class numbers, J. Riene Angew., Math. 337 (1982), 195-207.

4. Asymptotic behavior of number fields with prescribed l-class numbers, J. Number Theory 17 (1983), 191-203. 
5. __ An application of matrices over finite fields to algebraic number theory, Math. Comp. 41 (1983), 229-234.

6. 34.

7. F. Gerth and S. Graham, Application of a character sum estimate to a 2-class number density, J. Number Theory 19 (1984), 239-247.

8. L. Goldstein, Analytic number theor, Prentice-Hall, Englewood Cliffs, N. J., 1971.

9. G. Gras, Sur les l-classes d'idéaux dans les extensions cycliques relatives de degré premier I, Ann. Inst. Fourier (Grenoble) 23 (1973), 1-48; 23 (1973), 1-44.

10. (i. Hardy and E. Wright, An introduction to the theory of numbers (4th ed.), Oxford Univ. Press, London, 1965.

11. E. Inaba, Üher die Struktur der I-Klassengruppe zyklischer Zahlkörper von Primzahlgrad l, J. Fac. Sci. Imp. Univ. Tokyo, Sect. I 4 (1940), 61-115.

Di:partment of Mathematics, University of TeXas, Austin, TeXas 78712 